\title{
The next step for inpatient insulin dosing: using carbohydrate to insulin ratios
}

\begin{abstract}
Many out patients with diabetes have a successful history of self-adjusting their prandial (mealtime) insulin doses by counting the actual amount of carbohydrates they plan to consume and then individualizing the amount of their insulin dose accordingly. In contrast, hospitalized patients are typically prescribed doses of prandial insulin in advance, based on a predetermined amount of carbohydrate to be provided rather than individualized to the actual carbohydrate consumed.
\end{abstract}

Adjustment of prandial insulin doses is especially important for hospitalized patients because inconsistent food intake often occurs due to interruptions for tests, procedures and changes in appetite. Glucose results are less likely to be optimal when prandial insulin is administered without customized consideration for the ratio of pre-planned insulin doses to be delivered, and the patient's actual carbohydrate intake.

One way to optimize a match between the ideal insulin dose required and the actual foods consumed is to count the amount of carbohydrates before administering the insulin. Dosing insulin by carbohydrate counting is the standard of excellence for best practice in diabetes care. ${ }^{1}$ Many patients have mastered this essential component of diabetes self-management skills and appreciate the benefit of this practice by avoiding "serious complications of diabetes that may ensue when glycemic targets aren't achieved". 2

Keywords: carbohydrate counting- inpatient glycemic management, northridge hospital medical center, insulin, diabetes
Volume 2 Issue 2 - 2015

\author{
Debra Lesin Norman, Sandra Pieschel \\ Northridge Hospital medical center, USA \\ Valley Presbyterian Hospital, USA
}

\begin{abstract}
Correspondence: Debra Lesin Norman, Northridge Hospital medical center, 2280I Pera Rd Woodland Hills, California 91364, USA, Tel 3104224 61 I,Email debralesin@gmail.com
\end{abstract}

Received: July 22, 2014 | Published: March 24, 2015

\section{Demographics}

Northridge Hospital Medical Center (NHMC) is a 409 bed, notfor profit hospital located in the San Fernando Valley of Los Angeles County. The hospital serves adult and pediatric patients has a level II Trauma Center status. NHMC is a recipient of Health grades Distinguished Hospital Award for Clinical Excellence three years in a row and holds the Gold Seal of approval from The Joint Commission for Accreditation of Hospitals. Approximately one third of inpatients at NHMC have glycemic management concerns as analyzed by inpatient diagnostic codes compared daily census and pharmacy data of inpatients that are prescribed insulin. The 2011 community demographics for Los Angeles County provided by the California Department of Public Health showed 27.5\% of the local population has Diabetes. This increased significantly from 2007 data showing prevalence of $25.5 \%$.

\section{Introduction}

Inspired by the suggestion of several patients with Type 1 Diabetes, the Inpatient Interdisciplinary Insulin Committee recommended a process improvement initiative. The project would support an inpatient example of excellence for patients who are prescribed insulin. The evidence-based best practice model for prescribing insulin according to specific carbohydrate intake allows for individualized flexibility due to changes in lifestyle circumstances. Aligned with commonly accepted outpatient self-management practices, the calculation of insulin doses became based on actual intake of carbohydrate. Practices evolved, related to insulin administration within the hospital setting. The team collaborated toward successful inpatient integration of Carbohydrate to Insulin dosing for appropriate inpatients. This manuscript describes the structures and processes developed in this model.

\section{Appropriate inpatient target populations}

i. Type 1 Diabetes (Insulin Dependent)

ii. Type 2 Diabetes (Insulin Requiring)

iii. Steroid -Induced hyperglycemia (Insulin Requiring)

iv. Pregnant and requiring glycemic medication (Insulin Requiring)

\section{Disclosure statement}

This process improvement project was accepted for 2012 grant funding for Innovations in Practice awarded by Bayer Corporation. The funds were focused on training and educational materials such as posters, handouts and brochures, and were used to inspire staff morale and participation in the process changes proposed to be implemented. The $\$ 3,000$ Bayer Grant funds had no influence in study design or findings.

\section{Premise and purpose}

Widely accepted hospital Quality and Safety Indicators such as the Inpatient Core Measures, are benchmarks that track input measures of clinical practices. The Core Measures are based on science that shows certain therapeutic actions believed to be associated with optimal clinical and financial outcomes. The Core Measures do not require output measures to support change of practice. This paper describes a model of collaboration with inpatients that have the need or desire for involvement with their insulin dosing and self-management skills. 
It is the recommendation of the authors and the NHMC Inpatient Insulin Committee, that inpatient carbohydrate to insulin ratios for appropriate patients would be expected to lead to optimal glycemic control and optimal inpatient clinical and financial outcomes. This manuscript outlines the successful implementation of processes and structures at one facility, toward individualized insulin dosing aligned with best practice in the outpatient setting. The preliminary measures of success were noted in improvement by favorable satisfaction surveys and other less formal survey results of staff and patients.

\section{Overview}

\section{The dilemma of hospital meals}

Physiological bolus insulin regimens work well enough in a perfect world; however, hospital routines and hospital food are far from perfect. In reality, they are highly unpredictable. Meals are often interrupted for procedures and tests while patients' appetites vary with treatments, medications or pain. For inpatients, the variability of meals is further compromised by conditions related to the individual patient's acute illness, infection or injury.

In some hospitals, the food is not particularly palatable, or may be inconsistent with patients' preferences, resulting in patients not consuming what was provided according to pre-calculated nutritional needs. Assuming a patient will eat their prescribed meal is an unreliable benchmark, and can vary from meal to meal. It takes a conscientious and empowered nurse to keep insulin delivery safe and optimal. ${ }^{3}$

The recommended delivery of insulin in the hospital has evolved from an outdated use of reactive "sliding scales" toward a more proactive approach using more physiological analog insulin that integrates scheduled basal and adjustable prandial bolus dose regimens. ${ }^{4}$ Protocols for reacting to fluctuations in blood glucose have been used for years, and these "sliding scale" insulin regimens require measuring blood glucose levels several times each day and then administering enough insulin to cover above-normal values. However, the sliding scale approach makes no physiological sense and has been compared to giving sliding scale antibiotics to treat fever.

\section{Moving beyond traditional sliding scales}

Reacting to high glucose levels has the potential to lower glucose levels too much (Figure 1), after creating a physiological response that sends the glucose level higher than before, and can then lead to higher doses of insulin that further magnify the fluctuations. Reports found that increased length of hospital stay was associated with the use of sliding scale insulin protocols, prompting concern that it was harmful rather than helpful. ${ }^{5}$ By use of sliding scale insulin alone, the insulin dose is held until results are above target glucose levels. This method is less effective than scheduled basal and prandial insulin, adds variability and risk (Figure 1) and is associated with adverse outcomes. ${ }^{6,7}$

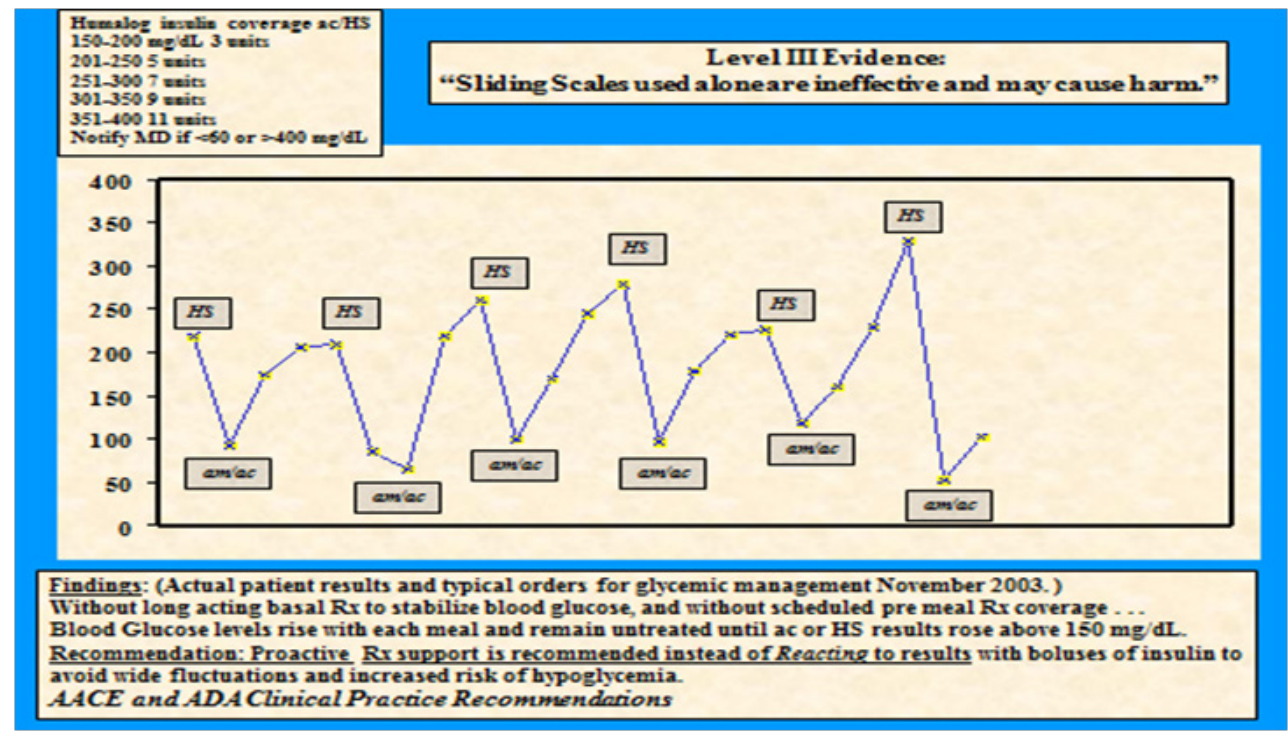

Figure I This picture illustrates the outdated, ineffective method of Sliding Scale Insulin used alone for many days without adjustment. Reactive fluctuations of glucose result in both hyperglycemia and hypoglycemia. This practice is a Class III Recommendation; the risk of sliding scale insulin used alone outweighs the benefit.

\section{Moving toward a chronic care model}

The chronic care model appreciates the role of the patient-centered approach to achieve glycemic goals. This proactive model accepted for outpatient best practice works effectively even in the acute care setting. Involvement of patients who already master this skill or who need to improve their self-management knowledge will support the collaborative chronic care model toward optimal prevention of complications. ${ }^{4}$

Physiological rapid acting analog insulin was first introduced in 1996 and made it possible to more effectively treat the distribution of metabolized carbohydrate foods. Non physiological Regular insulin first available in 1922, has an action curve that does not match the onset, peak or duration of nutritional metabolism. By comparison, the rapid acting analog insulin, when given at the start of the meal, has an action curve almost identical to food distribution. ${ }^{8}$

For many of us trained since 1998 when prandial analog insulin became available, the outdated, ineffective method of sliding scale dosing of insulin and/or use of non-physiological Regular insulin with meals are practices that we recognize as barriers to current clinical best practice standards as recommended by the American Diabetes Association, and the American Association of Clinical Endocrinologists ${ }^{9}$ and endorsed by The Society for Hospital Medicine. 
Prior to 1998 , we were traditionally taught to wait for blood glucose values to rise above $150 \mathrm{mg} / \mathrm{dl}$ before dosing a patient with insulin. Evidence grew that supported the association of decreased complications with better glycemic control. Support for changes in practice came from The Balanced Budget Act of 1997 which led to the initiation in 1998, of CMS/Medicare reimbursement for both Diabetes Self-Management Education and blood glucose monitors for home use. As patients were able to assess the effect of their insulin taken, the data clearly identified best practice as a self-management model.

For patients who are able to eat, the availability of glucometers provided immediate feedback that holding prandial insulin for results less than 130,140 or $150 \mathrm{mg} / \mathrm{dl}$, inherently leads to hyperglycemia and also greater potential for subsequent hypoglycemia. ${ }^{5}$ Sliding scales of subcutaneous insulin used alone is discouraged by the expert joint consensus panel of the American Diabetes Association and The American Association of Clinical Endocrinologists. ${ }^{9}$

On a graph assessment of the sliding scale, results will show a jagged, angular line rather than the linear graphic that reflects the desired steady state.

Sliding Scale insulin used alone has been consistently discouraged by AACE and ADA consensus statements since December 2003. Despite the overwhelming evidence to support individualized insulin management in the hospital setting, there is ongoing opportunity for education and training to overcome fear and misperceptions.

Although discouraged, it is not uncommon for sliding scale insulin to be ordered alone as we transition. Current evidence indicates that variability of blood glucose results in worse outcomes. ${ }^{5}$ The contemporary way to dose insulin is to ensure that blood glucose rarely rises or falls below the target range. When blood glucose values vary between hypoglycemia and hyperglycemia (below $70 \mathrm{mg} / \mathrm{dl}$ and/ or above $180 \mathrm{mg} / \mathrm{dl}$ ) the body is being stressed and many inflammatory and compensatory responses occur toward resuming homeostasis.

\section{Scheduled basal and prandial insulin bolus dosing}

A basal/bolus regimen allows us to replace the body's insulin the way the healthy pancreas would by normal physiological response mechanisms. The basal-bolus insulin regimens usually consist of basal analog insulin, such as Lantus (glargine) or Levemir (detemir), which are designed to distribute a fairly constant level of insulin over a 24 hour period. Basal insulin is prescribed toward achievement of glucose homeostasis directed at the liver's continuous release of endogenous glucose into the bloodstream. ${ }^{10}$ The bolus of rapid-acting analog insulin is delivered at mealtimes and is prescribed to process the food consumed.

\section{Supplemental correction insulin scales}

During administration of the bolus, corrections can be added to address out-of-target blood glucose results. These correction doses are often inaccurately referred to as a "sliding scales". When incorporated into a physiological insulin program, "correction scales" is a more appropriate descriptive term as they are intended to be supplemental to scheduled prandial doses.

\section{The prandial insulin bolus dose}

Exogenous prandial analog insulin is mealtime rapid acting insulin and may be provided as: Insulin Apidra, (glulisine), Novolog (aspart) or Humalog (lispro). Mealtime insulin administration is not just a task to check off the nurse's list, it is an opportunity for critical thinking. A nurse who delivers insulin is expected to have knowledge of insulin actions, onset, peak and durations which are particular to unique pharmacokinetics. This knowledge must be coupled with an understanding of how that insulin interacts with food. The best way to think about the pharmacokinetics of rapid-acting insulin is to understand that it was designed to mimic the pancreas as it secretes the hormone insulin into the bloodstream in response to carbohydrate. ${ }^{8}$

The healthy pancreas responds to the presence of glucose from food by secreting enough insulin to maintain a steady glucose level. As glucose is absorbed into the bloodstream from the digestive system, insulin levels would ideally raise and fall within the target range in response to changes in post prandial glucose levels.

\section{The patient-centered inpatient approach}

Best practice for experienced patients would be for the healthcare team to collaborate with them toward mutual agreement of options. Collaboration is associated with desirable glycemic outcomes and toward optimal patient satisfaction. ${ }^{2}$ In many cases, the best opportunity for success is optimal collaboration with the patient who is experienced in this self-management skill to promote optimal outcomes. Many patients and their families, already master this advanced skill with glucose self-management at home. Routinely, many of our inpatients with diabetes already master regular adjustments of their insulin doses to manage their glucose. Many adjust successfully during additional variations of physical activity. They also may be affected by unpredictable meals, emotional and physical stress factors. For patients who are not eating consistent amounts of carbohydrates, prescriptions for preset doses of mealtime insulin, is not a patient-centered approach. Changes in carbohydrate consumption require nurses to make real-time assessments of each patient's true needs and to act within the nurse's scope of practice to promote safety and efficacy.

Rapid-acting insulin has an onset of 15 to 30 minutes, peak action within 30 to 90 minutes and duration of action at 3-5hours. This insulin action curve has the closest approximation to glucose levels as carbohydrate is absorbed and processed in the bloodstream. ${ }^{11}$ When the pancreas is deficient of its release of insulin, relative to the patient's needs, the challenge is to mathematically determine how much insulin should be administered (Figure 2).

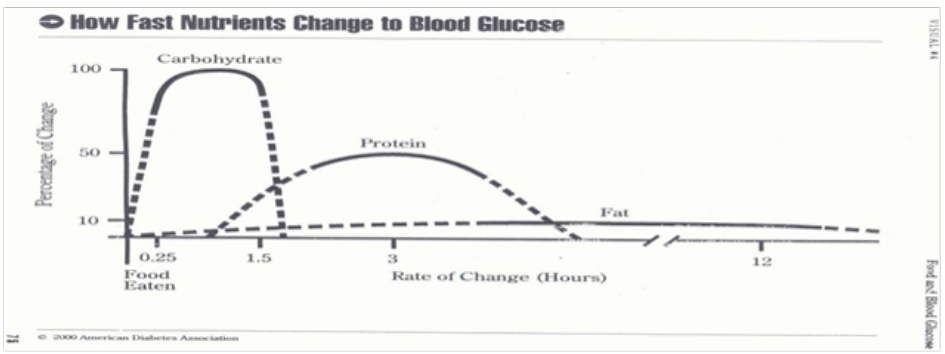

Figure 2 The American Diabetes Association's 2002 illustration of how carbohydrate, protein and fats distribute post meal glucose into the bloodstream along a timeline shown across the bottom of the picture. Most inpatient meals are served approximately 4-5hours apart. The action curves of rapid acting analog insulins were designed to match this digestion process. 
For nearly a century, since regular insulin was first available in 1922, we estimated the dose, hoped we reached targets and prayed that our patients did not have a hypoglycemic event. The fear of hypoglycemia has clouded our practice, making it common for patients to be closer to the higher, and more glucotoxic, insulinresistant range of the target zone. ${ }^{12,13}$

The fear of hypoglycemia often overshadows what we now understand to be the deleterious and insidious effects of hyperglycemia. Empirical data not only connects hyperglycemia to long-term complications, ${ }^{7}$ it also indicates that healing is slowed by the presence of excessive glucose. ${ }^{6}$ Additionally, the efficacy of medications is impaired when the chemistry of the blood is altered. ${ }^{14}$

\section{Patient benefits}

Despite all of this evidence, it remains a worthwhile challenge for nurses, patients and other team members to identify variations of need, estimate actual carbohydrates consumed and divide the number by the prescribed insulin ratio. By their example, clinicians can model and teach inpatients the skills of carbohydrate counting they will need to master as a self-management skill post discharge. Once patients learn to count carbohydrates and individualize that information to self-adjust their insulin doses to what they choose to eat. Patients can then be free to vary the amount of carbohydrate they consume and change a mealtime insulin dose to avoid hypoglycemia and/or excessive post prandial glucose excursions. This ability to vary food choices also promotes compliance. Our patients and staff were more successful with glucose management and we found it was associated with enhanced patient and staff satisfaction.

A concerned nurse might question whether such an approach would give patients the license to eat excessive quantities of food (Figure 3). For patients who would benefit from a limited calorie intake, that is indeed a valid concern. However, two concepts defeat this objection.

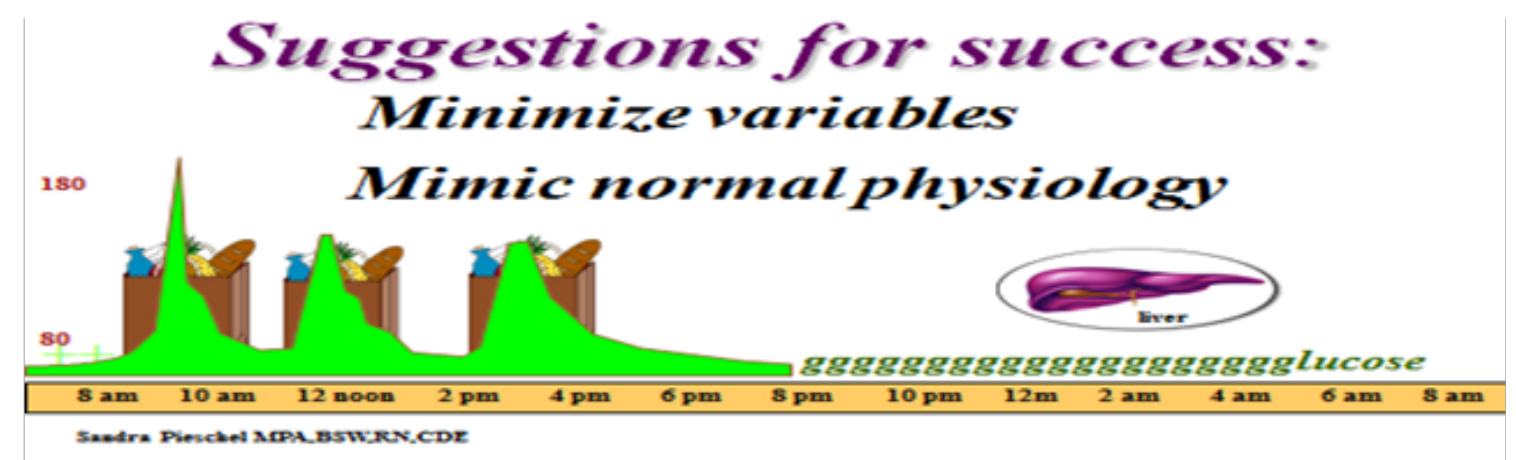

Figure 3 This illustration shows ideal insulin analogs given at the start of meals and matched to the distribution of glucose in the bloodstream post mealtimes anticipated along a timeline at the bottom. Glucose target levels are along the left axis shown in $\mathrm{mg} / \mathrm{dl}$.

A. By counting what the patient actually eats, the patient must pay attention to what they eat.

B. If the patient does veer from recommendations, why punish them with high glucose results?

Many clinical conditions integrate medication and nutritional recommendations as a combined therapeutic strategy, and do not result in potentially dire consequences when the dietary component of the regimen is not followed to ideals. For example, physicians prescribe blood pressure and statin medications in addition to dietary recommendations, yet those patients are not labeled as "noncompliant" when they eat outside of their prescribed diets. Teaching carbohydrate counting begins the foundation of best practice and removes some of the barriers to using insulin. There have been many reports in the existing literature that patients benefit from insulin therapy, and that delaying the use of insulin results in worse outcomes. ${ }^{15}$

When nurses collaborate with medical teams, they become better advocates for their patients, and indispensable champions as members of the health care team. Learning carbohydrate counting and working with the glycemic team's prescriber helps to avoid calls at mealtimes to adjust insulin, maintains compliance with nurses' scope of practice, and prevents out-of-target glucose results.

\section{There is no such thing as the "diabetic" or "ADA" diet}

The order for a "diabetic" or" ADA" diet itself can be confusing. Physicians traditionally order a calorie-limited ADA diet, unaware that the American Diabetes Association does not endorse any such diet, ${ }^{16}$ and that often a calorie-limited diet is inappropriate for the hospitalized patient. While a patient is ill or healing, a diet with decreasing caloric intake is not supported in the literature as therapeutic. ${ }^{17}$
The other concern regarding these traditional diet orders is that they must include a set amount of carbohydrates. This usually provides 60 grams of carbohydrate per meal for an $1800 \mathrm{kcal}$ diet divided into three meals of $600 \mathrm{kcals}$, with 270 calories of each meal (approximately 50\%) to be carbohydrate. This balanced diet is fine when the carbohydrate source is whole grains, fruits and vegetables. However, the reality in institutions is that the carbohydrate sources are often provided as refined breads, juice and empty calories.

Many patients complain when they order a high-protein or a healthy fat choice and it is removed from the order because it exceeds the caloric range, yet dessert is included. It is unfortunate when health economics prevent the modeling of healthy eating in the hospital. Fresh fruit, given its spoilage rate, is often replaced with fruit juice or canned fruit. Whole grain products are not the norm when they are cost prohibitive. There is also concern that limited caloric intake is not always appropriate for all patients. Many nurses have been known to remove juice from trays of patients who have diabetes because of the sugar content and empty calories when the grams of carbohydrate included in the juice are exactly what the doctor ordered.

\section{Practice at Northridge Hospital Medical Center (NHMC)}

At NHMC, we decided the best solution for our patients and for our nurses was carbohydrate counting and empowerment of nurses to make informed decisions at the bedside. Our order sets list both the ratios and the scheduled insulin doses based on a consistent carbohydrate diet. This menu routinely includes about 60 grams of carbohydrate per meal tray. Included in the order set is the usual carbohydrate to insulin ratio from which to select, and write-in space is provided for special circumstances. 


\section{How it works}

\section{Here is an example of one of our menus}

i. Grilled cheese sandwich (39grams CHO)

ii. Diet lemon lime soda (0grams CHO)

iii. Apple (15grams CHO)

iv. Tossed salad with ranch dressing and garbanzo beans (6grams $\mathrm{CHO})$

v. The total amount of carbohydrate is 60 grams and the prescribed order is for 6units of insulin for each meal. This formula represents a ratio of 1 unit of insulin for each 10 grams of $\mathrm{CHO}$.

What a patient might actually eat

i. Half sandwich (20grams $\mathrm{CHO})$

ii. Diet soda (0grams CHO)

iii. If 1 unit of prandial insulin is to be delivered for each 10 grams $\mathrm{CHO}$ consumed, then for the $20 \mathrm{grams}$ of $\mathrm{CHO}$ which is actually consumed, the patients should receive 2 units of insulin.

With carbohydrate to insulin dosing ratios, the nurse can then appropriately administer 2 units of insulin, which is a much safer dose than preset 6 units prescribed. Our practice would avoid an unintended overdose of insulin which could result in post-prandial hypoglycemia. Added to the calculation would be a supplemental correction scale for the current blood glucose result. This is when the patient's glucose level can be addressed if it is above $150 \mathrm{mg} / \mathrm{dl}$. The nurse will refer to the prescribed correction scale and add those insulin units into the adjusted prandial dose to provide one prandial injection. Our correction scales are labeled mild coverage for the insulinsensitive patient, moderate coverage for the average patient, and high coverage for the insulin-resistant patient. Prescribers can also design customized correction scales relative to each patient's personalized insulin sensitivity.

In our same patient example will be 2 units for the food intake (20grams CHO) and to address the glucose result of $175 \mathrm{mg} / \mathrm{dl}$, an additional unit will be added according to the correction scale. The three unit adjusted dose should return the hyperglycemia into the target range. Confirmation of the prescription will be at the next blood glucose check. The best time to assess the effect would be two hours after the first bite of the meal. However, inpatient care teams are typically busy and possible confusion about re-dosing supported checking glucose only when insulin was to be administered at the next meal. Adjustments to the prescription should be made if a larger than $50 \mathrm{mg} / \mathrm{dl}$ fluctuation resulted between the before and after meal glucose values.

\section{Timing of delivery}

An important consideration is when to deliver the insulin. The rapid-acting analog insulins aspart, lispro and glulisine were designed to mimic the kinetics of the glucose absorption from the gut. These insulins are most effective when administered immediately prior to the meal. We prefer to give the insulin once the tray has arrived and the patient confirms that they plan to eat the entire meal. Experience has taught us to be a bit more flexible. On our pediatric floors, we allow children 20-30minutes to eat their carbohydrate containing food, and then inject insulin doses based upon what was actually eaten. If a child has not finished his or her treats and we expect them to do so, we may cover the carbohydrates proactively. For adults, it is left to nursing judgment. Our order set offers flexibility as it reads, "administer just prior to the meal or for patients with questionable intake, within 20minutes from the start of the meal." For patients with gastroparesis (delayed gastric emptying) or for those with a history of nausea and vomiting, orders can be obtained to wait longer to avoid mismatches in dosing.

\section{Hospital systems to Support Carbohydrate (CHO) to Insulin Ratios (CIR)}

To support our practice, we needed several structures and processes in place.

i. Insulin order sets were redesigned

ii. Carbohydrate amounts were listed on tray tickets

iii. Tray delivery times were coordinated between dietary and nursing

iv. Music was played to alert staff that trays were being delivered

v. Patients and staff were educated on carbohydrate counting and

vi. Brochures were developed to market and promote the program

vii. Posters of the process were placed in available visible locations

viii. Tray tents to identify these patients were placed on meal trays

ix. Handouts were provided to support the program

$\mathrm{x}$. Educational videos encouraged reinforcement on patient televisions

\section{Training and preparation for process changes}

Staff training began very early in the process. When a patient who carbohydrate counted at home was admitted to the hospital, their ratios were handwritten on the order set and just-in-time teaching was provided to the staff. Dietary and educational staff was educated by case study presentations at unit meetings. A brochure was developed to help the staff implement critical thinking throughout the process, and our poster campaign kept staff informed of the benefits of carbohydrate counting. Trays delivered to insulin-using patients are covered with a green warming dome to distinguish them from patients who did not need glucose monitoring and insulin. The census board identified patients with glycemic management concerns to alert nursing and dietary staff.

\section{The rewards}

It took special effort to change practice, and the rewards we have experienced are worth it. Consistent with outside evidence, initial NHMC surveys (2012 $n 18$ patients and 34 staff satisfaction reports)

i. Feedback was overwhelmingly positive from patients with Type 1 diabetes.

ii. Patients' feedback expressed consistent appreciation for involvement with management of their glucose levels while in the hospital as at home.

iii. They communicated the positive impression of the hospital when staff individualized results for specific patient needs.

iv. Once discharged, patients reported they felt more prepared to self-manage at home 
v. Our inpatients feel they are better prepared to leave the hospital with a greater understanding of the variable management of their disease.

vi. Recognition of pride that NHMC practice reflects current best practice in the science and art of diabetes care.

\section{Summary}

For appropriate inpatients, consideration of carbohydrate to insulin dosing ratios is encouraged as a universal practice to be available as a patient option at NHMC. These self-management input efforts are aligned with best practice principles. ${ }^{4}$ Collaboration toward optimal glycemic results is expected to improve clinical and financial outcomes, improve compliance and satisfaction for both patients and staff (UKPDS). Ongoing collection and analysis of satisfaction surveys, readmissions, length of hospital stay, hypoglycemia and hyperglycemia trends will determine whether integration of this outpatient best practice model when used for inpatients will provide the anticipated positive results and future benefit as recognized in the outpatient setting.

\section{Acknowledgments}

a) The nursing staff at Northridge Hospital Medical Center who accepted the new concepts with grace and vigor

b) The physician staff who encouraged practice change and helped lead the charge

c) Our patients who patiently taught our staff how diabetes is managed in the non acute world

d) Bayer Healthcare "Innovations in Practice" grant for funding and inspiring innovation

e) Chris Dean Elliot for his translation from diabetes talks to human communication.

\section{Conflict of interest}

Author declares that there is no conflict of interest.

\section{References}

1. DAFNE Study Group. Training in flexible, intensive insulin management to enable dietary freedom in people with type 1 diabetes: dose adjustment for normal eating (DAFNE) randomized controlled trial. BMJ 2002;325(7367):746-751.

2. Holman RR, Paul SK, Bethel MA, et al. 10year follow-up of intensive glucose control in Type 2 diabetes. $N$ Engl $\mathrm{J}$ Med. 2008;359(15):1577-1589.
3. McKnight K, Carter L. From Trays to Tube Feedings: Overcoming the Challenges of Hospital Nutrition and Glycemic Control. Diabetes Spectrum. 2008;21(4):233-240.

4. Seley J, Weinger K. The State of the Science on Nursing Best Practices for Diabetes Self-Management. American J Nursing. 2007;107(6):73-78.

5. Sawing G, Shaughnessy A. Glucose Control In Hospitalized Patients. Am Fam Physician. 2010;81(9):1121-1124.

6. The UK Prospective Diabetes Study Group (UKPDS 33). Intensive blood-glucose control with sulphonylureas or insulin compared with conventional treatment and risk of complications in patients with Type 2 diabetes. Lancet. 1998;352(9131):837-853.

7. The Diabetes Control and Complications Trial (DCCT) Research Group. The effect of intensive treatment of diabetes on the development and progression of long-term complications in insulin-dependent diabetes mellitus. N Engl J Med. 1993;329(14):977-986.

8. Noble SL, Johnstone E, Walton B. Insulin lispro: A Fast Acting Insulin analog. Am Fam Physician. 1998;57(2):279-286.

9. Moghissi ES1, Korytkowski MT, DiNardo M, et al. (AACE/ADA) American Association of Clinical Endocrinologists and American Diabetes Association Consensus Statement on Inpatient Glycemic Control. Diabetes Care. 2009;32(6):1119-1131.

10. Aronoff LS, Berkowitz K, Shreiner B, et al. Glucose Metabolism and Regulation: Beyond Insulin and Glucagon. Diabetes Spectrum. 2004;17(3):183-190.

11. National Diabetes Information Clearing house (NDIC): A service of the National Institute of Diabetes and Digestive and Kidney Diseases (NIDDK) National Institutes of Health (NIH), USA.

12. Cryer PE. Banting Lecture. Hypoglycemia: the limiting factor in the management of IDDM. Diabetes. 1994;43(11):1378-1389.

13. Cryer PE. Hypoglycemia risk reduction in type 1 diabetes. Exp Clinical Endocrinology Diabetes. 2001;109(Suppl 2):S412-423.

14. Karci A, Tasdogen A, Erkin Y, et al. The analgesic effect of morphine on postoperative pain in diabetic patients. Acta Anaesthesiologica Scandinavica. 2004;48(5):619-624.

15. American Diabetes Association. Translation of the diabetes nutrition recommendations for health care institutions (Position Statement). Diabetes Care. 2002;25(Suppl 1):S61-S63.

16. American Diabetes Association. Evidenced-based nutrition principles and recommendations for the treatment and prevention of diabetes and related complications (Position Statement). Diabetes Care. 2002;25(Suppl 1):S50-S60.

17. Goodall G, Sarpong EM, Hayes C, et al. The consequences of delaying insulin initiation in UK type 2 diabetes patients failing oral hyperglycaemic agents: a modeling study. BMC Endocr Disord. 2009;9:19. 